\title{
Numerical Analysis on Cold Crucible Using 3D H- $\phi$ Method and Finite Volume Method with Non-staggered BFC Grid System
}

\author{
Pil-Ryung CHA, Yun-Seong HWANG, Yung-Joo OH, ") Soon Hyo CHUNG ${ }^{1)}$ and Jong-Kyu YOON \\ Department of Metallurgical Engineering, Seoul National University, Seoul 151-742, Korea. \\ 1) Division of Metals, Korea Institute of Science and Technology, P.O. Box 131, Cheongryang, Seoul 130-650, Korea.
}

(Received on January 16, 1996; accepted in final form on April 12, 1996)

\begin{abstract}
Generally, numerical analysis of MHD systems including cold crucible requires much amounts of calculating resources. These systems often include 3D electromagnetic field, fluid flow in irregular boundaries, solidification, even coupling between electromagnetic field and fluid flow. Two kinds of basically different simulation techniques are necessary for effective calculation of these MHD systems. These are FEM (Finite Element Method) for calculation of electromagnetic field and FVM (Finite Volume Method) with BFC (Body Fitted Coordinate) for fluid flow. But many researchers have been tried to solve these problems by other methods because the use of the combined method consumes large quantity of memory and computing time. Most of numerical models on cold crucible do not include the analysis of fiuid flow. For calculation of electromagnetic field, 2D axisymmetric wire model, it's improved model or Boundary Element Method have been widely used instead of fully 3D FEM.

In this study, 3D H- $\phi$ formulation for electromagnetic field by FEM and a technique using non-staggered grid system for fluid flow by FVM with BFC were employed to save the memory space and calculation time in numerical analysis of cold crucible. A package of numerical models including electromagnetic, fluid dynamic, heat transfer and solidification model was constructed and applied to the numerical simulation of cold crucible. Validity of the electromagnetic model was confirmed by comparison between the results from calculation and those from direct measurement. Verification of the developed code on fluid dynamic calculation was carried out by its comparison with the commercial code PHOENICS. Influence of some important operating parameters on the meniscus shape and solidification front were investigated using the developed package. Temperature distribution in the molten tin was uniform because of the circulating flow induced by non-uniform distribution of electromagnetic force and the heat transfer through mold wall at the melt-mold contacted region was noticeably reduced as a result of magnetic pressure.
\end{abstract}

KEY WORDS: cold crucible; MHD; electromagnetic field; fluid flow; FEM; BFC; non-staggered grid.

\section{Introduction}

Cold crucible is composed of several water cooled conducting segments and axisymmetrically arranged coils which carry high frequency current. This crucible has slits between segments. These slits play a role on penetrating magnetic field to charge. Use of cold crucible has been widespread for the melting and continuous casting of metals with high melting points or are very active with refractories.

Like other MHD systems, theoretical studies are very important for the optimization of the design of cold crucible and the choice of the appropriate operating conditions of casting. However, there are several nontrivial problems in developments of mathermatical model for cold crucible. Followings are the representative ones.

- Geometry of crucible is three dimensional.

- Charge has free boundary which interacts mutually with magnetic field and also has solidification phenomenon.

- Fluid flow with irregular boundary must be considered to estimate heat transfer.

Because of these difficulties, most numerical analysis researches on cold crucible have dealt with a partial aspects of overall phenomena. ${ }^{1-3)}$ Most of the studies do not include analysis of fluid flow and solidification phenomena. For the calculation of electromagnetic field, 2D axisymmetric model has been frequently used. 2D axisymmetric wire model and improved model considering the effects of slits assuming the conductor as a bundle of axisymmetric conducting wires have been widely used as 2D axisymmetric models. ${ }^{4-8)}$ Boundary Element Method and FEM also has been used. ${ }^{2,3,7)}$ Iwai et al. calculated electromagnetic field considering three dimensional geometry of cold crucible by using BEM. ${ }^{7)}$ Integrated analysis on overall phenomena was conducted by Tanaka et al. ${ }^{9)}$ In their research, magnetic pressure was calculated by boundary integral method by assuming $2 \mathrm{D}$ axisymmetric model. $2 \mathrm{D}$ axisymmetric model has advantages in saving the memory space and calculation time but its application is limited to cold crucible with relatively simple geometry. 
In this study, an efficient numerical analysis program capable of calculating 3D electromagnetic field in cold crucible and liquid metal flow with heat transfer in a domain confined by a free surface and a solidification front was developed. Two newly suggested numerical techniques are introduced to reduce computational comsumption. One of them is 'H- $\phi$ method' ${ }^{10)}$ which uses three kinds of variables $\left(H_{x}, H_{y}, H_{z}\right)$ in conductor and only one variable $(\phi)$ in non-conducting region in calculating the electromagnetic field. The other is pressure gradient interpolation method developed to estimate fluid flow by FVM which uses boundary fitted nonstaggered grid system. By this way, the amounts of memory size required can be reduced to about half or one third as small as the case of staggered grid system. To avoid checkerboard phenomenon ${ }^{11)}$ which occurs often in non-staggered grid system, pressure gradient interpolation method ${ }^{12)}$ was applied. The electromagnetic field distribution, the meniscus shape of charge, Joule heating and electromagnetic body force in charge could be calculated by the complete three dimensional electromagnetic analysis of cold crucible. With the numerical data obtained from the FEM calculation for electromagnetic field and BFC-FVM code "MPLGC" developed by Materials Division at Seoul National University, fluid flow field and solidification phenomenon have been investigated.

The computational analysis was confirmed by comparing with the results of the experimental data for tin system. And then a series of calculation has been conducted to investigate the effects of main process parameters.

\section{Numerical Analysis Method}

Schematic structure of the whole process of numerical analysis is shown in Fig. 1. The process is composed of three kinds of calculation processes; they are calculations of electromagnetic field, fluid flow field and heat transfer. Shape calculation is interrelated with electromagnetic field calculation process. In addition, post processor and convergence monitoring system are also a part of the whole process. These processes are mutually coupled through the interactions of electromagnetics, fluid dynamics and heat transfer. The calculation domain of this cold crucible system is wedged half section of a segment including a free surface of melt and a solidification front, which must be determined through an interaction among the processes.

\subsection{Calculation of Electromagnetic Field}

\subsubsection{Governing Equations}

In an electromagnetic system including conducting fluid, the electric field intensity $E$, the magnetic field intensity $H$, and the magnetic flux density $B$ are described by Maxwell's equations. From the Maxwell's equations, the following equations which are needed to solve the electromagnetic field region in a cold crucible are given:

$$
\begin{array}{r}
\nabla \times H_{0}=J_{0} \\
\nabla \times H_{r}=\sigma E \\
\nabla \times E=-j \omega \mu\left(H_{0}+H_{r}\right) \\
\nabla \cdot\left(H_{0}+H_{r}\right)=0
\end{array}
$$

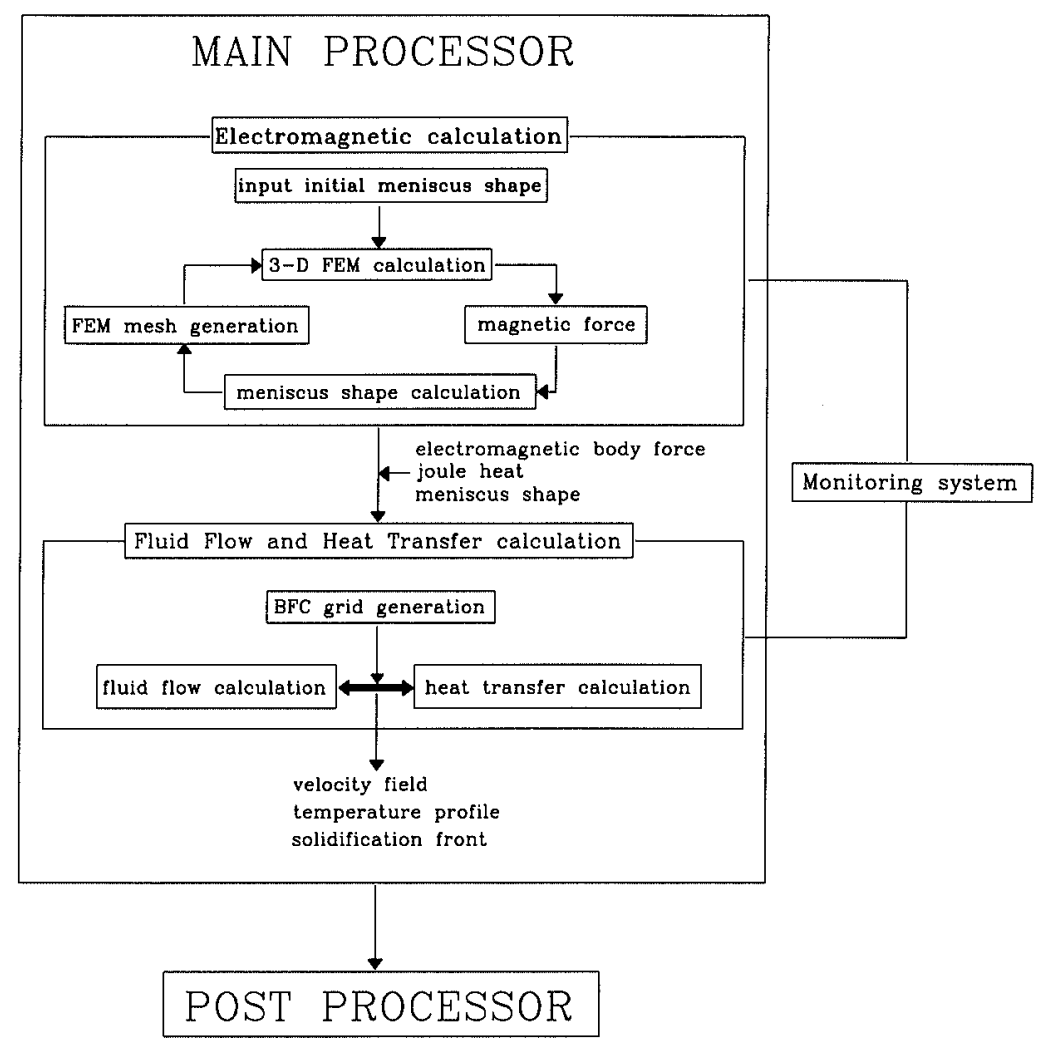

Fig. 1. Flow chart of calculation. 
In a free space where the induction coil is present, $\nabla \times$ $H=\nabla \times\left(H_{0}+H_{r}\right)=\nabla \times H_{0}=J_{0}$ and consequently $\nabla \times$ $H_{r}=0$. This means that $H_{r}$ can be described as a gradient of a scalar because curl of grad of a scalar is always zero for any scalar. The reduced magnetic scalar potential $\phi$ can be defined as;

$$
H_{r}=-\nabla \phi
$$

$\phi$ has been adopted as the variable in the free space in the present study to save calculation time significantly. ${ }^{14)}$

\subsubsection{Variational Equation}

Details of variational formulations are described in Ref. 13). By multiplying a vector weighting function $W$ on both sides of Eq. (3) and taking volume integral, variational equation within conductor is stated as;

$$
\begin{array}{rl}
\int_{V_{\mathrm{c}}} & j \omega \mu W \cdot\left(H_{0}+H_{r}\right) d V \\
\quad= & \int_{V_{\mathrm{c}}}\left(\frac{1}{\sigma}\right)\left(\nabla \times H_{r}\right) \cdot(\nabla \times W) d V-\int_{S} W \cdot\left(E \times n_{\mathrm{c}}\right) d S
\end{array}
$$

By multiplying the product of a scalar trial function $w$ and $j \omega \mu$ on Eq. (4), and by taking volume integral, it is written as;

$$
\int_{V_{\mathrm{nc}}} j \omega \mu w \nabla \cdot\left(H_{0}+H_{r}\right) d V=0
$$

Using vector identity and Eqs. (3) and (5), and applying Gauss theorem, variational equation within non-conducting free space is written as;

$$
\int_{V_{\mathrm{nc}}} j \omega \mu\left(\nabla w \cdot \nabla \phi-\nabla w \cdot H_{0}\right) d V+\int_{S} \nabla w \cdot\left(E \times n_{\mathrm{nc}}\right) d S=0
$$

At the boundary between the conducting and nonconducting regions, the unit outward normal vectors $n_{\mathrm{c}}$ and $n_{\mathrm{nc}}$ have opposite signs, i.e. $n_{\mathrm{c}}=-n_{\mathrm{nc}}$ and trial functions are defined as $W=-\nabla w$ to eliminate surface integral terms. From variational formulation within conductor and variational formulation in non-conducting free space, the overall variational equation is obtained as follows:

$$
\begin{aligned}
\int_{V_{\mathrm{nc}}}(\nabla w \cdot \nabla \phi) d V & +\int_{V_{\mathrm{c}}}\left(\frac{1}{j \omega \mu \sigma}\right)\left(\nabla \times H_{r}\right) \cdot(\nabla \times W) d V \\
& +\int_{V_{\mathrm{c}}} W \cdot H_{\mathrm{r}} d V \\
=\int_{S} w H_{0} \cdot n_{\mathrm{nc}} d S & -\int_{V_{\mathrm{c}}} W \cdot H_{0} d V \quad \ldots \ldots \ldots \ldots \ldots \ldots . . . \ldots(9)
\end{aligned}
$$

where, $\quad W=-\nabla w$ on $S$.

Equation (9) is the so-called $\mathrm{H}-\phi$ formulation. In the above equation the only vector variables exist in conducting region and the only scalar variables exist in non-conducting free space. So, the number of variables at nodal point in conducting region is three and that in non-conducting free space is one. The edge finite element method is adopted in conducting region, and the nodal finite element method in non-conducting free space. The $3 \mathrm{D}$ region is divided into cubic elements, and the stiffness matrix is solved by the Crout method.

\subsection{Determination of the Shape}

The shape of meniscus is calculated as follows:

(1) The magnetic field is calculated for the initial shape of the charge by 3D FEM model.

(2) The magnetic pressure $P_{\mathrm{m}}$, hydrostatic pressure $P_{\mathrm{g}}$, and pressure due to surface tension $P_{\mathrm{s}}$ on the surface of melt are calculated as given by the following equations.

$$
\begin{array}{r}
P_{\mathrm{m}}=\frac{\mu H_{\tau}^{2}}{2} \ldots \\
P_{\mathrm{g}}=\rho g z \quad \ldots . \\
P_{\mathrm{s}}=\Gamma\left(\frac{1}{R_{1}}+\frac{1}{R_{2}}\right)
\end{array}
$$

(3) The sum of the above three pressures on surface is obtained at every nodes on meniscus as the following equation.

$$
P_{\mathrm{m}}+P_{\mathrm{g}}+P_{\mathrm{s}}=\mathrm{constant}
$$

By comparison of this sum of every nodes, the displacement of meniscus is determined and a new shape of meniscus is calculated based on this displacement.

(4) Iteration is repeated with new shape until the displacement becomes less than a convergence criterion.

(5) If convergence is reached the shape of meniscus is saved in file and electromagnetic force and Joule heating are calculated for next step of oveall simulation.

The electromagnetic force $F$ is obtained by the following equation.

$$
F=J_{\mathrm{e}} \times B
$$

The Joule heat per unit volume $Q$ is calculated using the following equations:

$$
Q=\frac{\left|J_{\mathrm{e}}\right|^{2}}{\sigma}
$$

where,

$$
J_{\mathrm{e}}=\nabla \times H_{r} .
$$

It is then volume integrated for charge, cold crucible and induction coil, respectively.

\subsection{Calculation of Velocity Field}

Fluid flow phenomena occur in molten region of the charge and there is an irregular boundary of free surface in the charge. For such a problem, the FVM with a boundary fitted curvilinear coordinate system is the appropriate method. The governing equation of continuity and momentum in non-orthogonal body-fitted curvilinear coordinate for an incompressible Newtonian fluid are writen by general conservative form. ${ }^{15}$ )

$$
\begin{array}{r}
\frac{1}{J}\left\{\frac{\partial}{\partial \xi_{i}}\left(\rho G^{i}\right)\right\}=0 \\
\frac{1}{J}\left\{\frac{\partial}{\partial \xi_{i}}\left(\rho G^{i} u_{i}\right)\right\}=\frac{1}{J}\left\{\frac{\partial}{\partial \xi_{i}} \Gamma_{\phi} J\left(g^{i j} \frac{\partial u_{i}}{\partial \xi_{j}}\right)\right\}+S_{u_{i}} \ldots \ldots . . . \ldots \ldots . . . . .
\end{array}
$$




$$
S_{u_{i}}=-\frac{\partial p}{\partial x_{i}}+\frac{\partial}{\partial x_{j}}\left(\mu_{i} \frac{\partial u_{j}}{\partial x_{i}}\right)+\left(J_{j} B_{k}-J_{k} B_{j}\right)
$$

where, $\quad G^{i}=J \frac{\partial \xi_{i}}{x_{j}} u_{j}, \quad g^{i j}=\frac{\partial \xi_{i}}{\partial x_{k}} \frac{\partial \xi_{j}}{\partial x_{k}}$.

In this formulation, covariant velocity vectors are used as main variables. The last term in the right hand side of Eq. (18) includes the time averaged electromagnetic body force which is determined by the foregoing electromagnetic calculation by FEM.

The solution of Navier-Stokes equations by FVM requires that the velocities used to satisfy the continuity equation should also simultaneously satisfy the momentum equations. Well-known staggered grid system explicitly satisfy this requirement systematically. ${ }^{11)}$ However, it consumes large amounts of memory space because of its various complex geometric variables. To avoid this difficulty, the method of non-staggered grid system is adopted. This method could reduce the number of geometric variables to about one third of staggered grid system. To cure the checkerboard phenomenon of pressure which is the major problem of non-staggered grid system the pressure gradient interpolation method ${ }^{12)}$ is applied. So far, momentum interpolation method of Rhie and Chow ${ }^{16)}$ has been applied to cure the checkerboard phenomena. However, recently proposed pressure gradient method is more convenient to deal with and saves more memory space. Besides of this, recently developed MCGS (Modified Conjugate Gradient Sover) ${ }^{17)}$ is used to improve the convergence of calculation of pressure field.

In this study, $k-\varepsilon$ turbulent model ${ }^{18)}$ is used. Viscosity and Diffusion coefficient appeared in Eq. (17) means the enhanced quantity by turbulent eddy motion described as follows.

$$
\mu_{\text {eff }}=\mu_{1}+\mu_{\mathrm{t}}
$$

where effective viscosity $\mu_{\mathrm{cff}}$ is the sum of laminar viscosity $\mu_{1}$ and turbulent viscosity $\mu_{\mathrm{t}} \cdot \mu_{\mathrm{t}}=\rho l^{2}|d u / d y|=$ $\rho C_{\mu}\left(k^{2} / \varepsilon\right)$ from $k-\varepsilon$ model, where $l, k, \varepsilon$ and $C_{\mu}$ are mixing length, turbulent kinetic energy, turbulent dissipation energy and constant $(=0.09)$ respectively.

\subsection{Calculation of Heat Transfer and Solidification}

The governing equation of heat transfer in general coordinate is given by Eq. (20). ${ }^{15)}$

$$
\begin{gathered}
\frac{1}{J}\left\{\frac{\partial}{\partial \xi_{i}}\left(\rho G^{i} T\right)\right\}=\frac{1}{J}\left\{\frac{\partial}{\partial \xi_{i}} \Gamma_{T} J\left(g^{i j} \frac{\partial T}{\partial \xi_{j}}\right)\right\}+S_{T} \ldots \\
S_{T}=\frac{J_{\mathrm{e}}^{2}}{\sigma C_{p}}
\end{gathered}
$$

where $\Gamma_{T}$ is $\mu / \operatorname{Pr}+\mu_{\mathrm{t}} / \sigma_{\mathrm{t}}, \sigma_{\mathrm{t}}$ is turbulent prandtl number and $C_{p}$ is specific heat, respectively.

The last term in the right hand side of Eq. (20) includes the time averaged Joule heating obtained as one of the results from the foregoing FEM calculation. For the boundary condition, radiation, conduction and adiabatic condition are considered. For symmetric planes and planes contacting with ceramic pedestal, adiabatic con- dition is applied. The radiative heat transter is considered at the free surface and heat conductions are considered where charge and crucible wall are contacting each other. The following is a combined boundary condition for heat transfer.

$$
q=h\left(T-T_{0}\right)+\varepsilon \sigma\left(T^{4}-T_{0}^{4}\right)
$$

where $h, \varepsilon, \sigma$ are heat transfer coefficient, emissivity and Stefan-Boltzmann constant, respectively.

To obtain heat transfer coefficient $h$ in the right hand side of Eq. (21), the temperature of cooling water was measured. The method of calculation used to obtain $h$ is as following.

$$
q=-\left.k \frac{d T}{d x}\right|_{\text {charge surface }}=h\left(\mathrm{~T}_{\text {charge surface }}-T_{\text {crucible }}\right)
$$

$$
-k \frac{T_{\text {charge surface }}-T_{\text {inner node }}}{\Delta x}=h\left(T_{\text {charge surface }}-T_{\text {crucible }}\right)
$$

$$
h=-k \frac{T_{\text {charge surface }}-T_{\text {inner node }}}{\Delta x\left(T_{\text {charge surface }}-T_{\text {crucible }}\right)}
$$

where $x, \Delta x, T_{\text {charge surface, }} T_{\text {inner node }}$ and $T_{\text {crucible }}$ are radial direction length, differentiated length of $x$ in the control volume of surface of charge, temperature of charge surface, temperature of first inner node from charge surface and temperature of crucible, respectively.

In Eq. (21-c), $T_{\text {charge surface }}$ and $T_{\text {inner node }}$ can be obtained from previous iteration step. $T_{\text {crucible }}$ was set equal to temperature of cooling water which could be obtained by measurement. Heat transfer coefficient $h$ obtained by previous process is inserted to Eq. (21) to complete boundary condition.

Physical properties of Tin used in the calculation are shown in Table 1. Effective heat capacity method ${ }^{19)}$ is used for solidification modeling. In this study, there is

\begin{tabular}{|c|c|c|}
\hline Melting temperature & $T_{\mathrm{m}}$ & $231.9^{\circ} \mathrm{C}$ \\
\hline $\begin{array}{l}\text { Specific heat at room } \\
\text { temperature }\end{array}$ & $C_{p}$ & $240 \mathrm{~J} \mathrm{~kg}^{-1} \mathrm{~K}^{-1}$ \\
\hline $\begin{array}{l}\text { Latent heat during } \\
\text { solidification }\end{array}$ & $L$ & $59.6 \mathrm{~J} / \mathrm{kg}$ \\
\hline Density & $\rho$ & $7000 \mathrm{~kg} / \mathrm{m}^{3}$ \\
\hline Thermal conductivity & $k$ & $31 \mathrm{~kg} \cdot \mathrm{m} \cdot \mathrm{sec}^{-1} \mathrm{~K}^{-1}$ \\
\hline Surface tension & $r$ & $0.544 \mathrm{~N} / \mathrm{m}$ \\
\hline $\begin{array}{l}\text { Laminar viscosity in } \\
\text { liquidus }\end{array}$ & $\mu$ & $\begin{array}{c}1.85 \times 10^{-3} \mathrm{~N} \cdot \mathrm{sec} / \mathrm{m}^{2} \\
\quad:\end{array}$ \\
\hline $\begin{array}{l}\text { Thermal expansion } \\
\text { coefficient }\end{array}$ & $\beta$ & $0.875 \times 10^{-4}$ \\
\hline Electric conductivity & $\sigma$ & $9.01 \mathrm{MS} / \mathrm{m}$ \\
\hline Emissivity & $\varepsilon$ & $0.32-0.6$ \\
\hline
\end{tabular}
nearly no mushy region because pure tin is used as melt. Therefore, mushy region is sufficiently suppressed to be very thin.

\section{Simulation Condition of the System}

The system of a cold crucible is composed of a eightturned water cooled coil with $7 \mathrm{~mm} \times 7 \mathrm{~mm}$ square cross

Table 1. Physical properties of tin used in the calculation. 


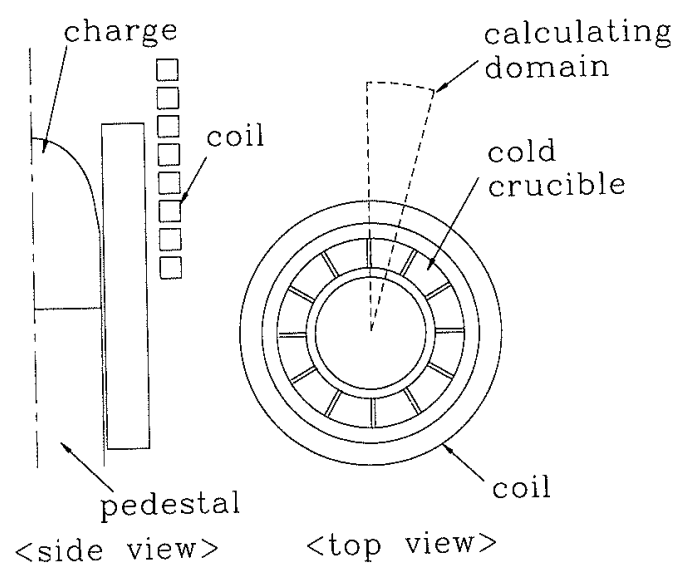

Fig. 2. Schematic diagram of calculating domain.

section and a water cooled crucible made of copper with $40 \mathrm{~mm} \phi$ of I.D., $76 \mathrm{~mm} \phi$ of O.D. and $150 \mathrm{~mm}$ of height was used in experiments. A continuous casting type straight crucible has 12 segments with the slit width of $1.5 \mathrm{~mm}$ periodically arranged along the circumferential direction of the crucible. Considering the periodical arrangement of the segments, only an half portion of the one segment is enough for calculation of electromagnetic field. The shape of cold crucible is three dimensional. Figure 2 shows the whole geometry of the cold crucible and the domain used in the calculation.

In calculation of fluid flow field, circumferential directional velocity is neglected. The electromagnetic field is explicitly three dimensional but fluid flow field is conditionally two dimensional. In this study, frequency of electric current is sufficiently high so that the circumferential deviation of magnetic forces is small.

\section{Verification}

Three courses of calculation were conducted. They are validation of electromagnetic field calculation and dynamic calculation, and calculation to investigate influence of main parameters such as electric current and frequency. Computations were carried out mainly at AlphaStation 250 4/266 and 200 4/233 of DEC (Digital Equipment Cooporation).

\subsection{Verification of Calculation of Electromagnetic Field}

To verify accuracy of the calculation results, distribution of the magnetic flux density in an empty cold crucible was measured at the apparatus with the same geometry for the calculation. Maximum power and frequency range of the generator are $15 \mathrm{~kW}$ and $5-9.6 \mathrm{kHz}$, respectively. A miniature induction sensor coil made of $0.1 \mathrm{~mm} \phi$ enameled copper wire wound around a glass rod of $1 \mathrm{~mm} \phi$ was used to measure the vertical component of the magnetic flux density $B_{z}$. To minimize the electrical noise during measurement, the leading wires of the sensor coil were closely twisted and shielded by a copper tube. A 3D translation device was used to position the sensor coil at each measure point. The induced $\mathrm{AC}$ potential in the sensor coil was measured with a high impedance digital voltmeter. Assuming that the magnetic flux density is uniform within the sensor coil, it can be calculated from the following relationship based on

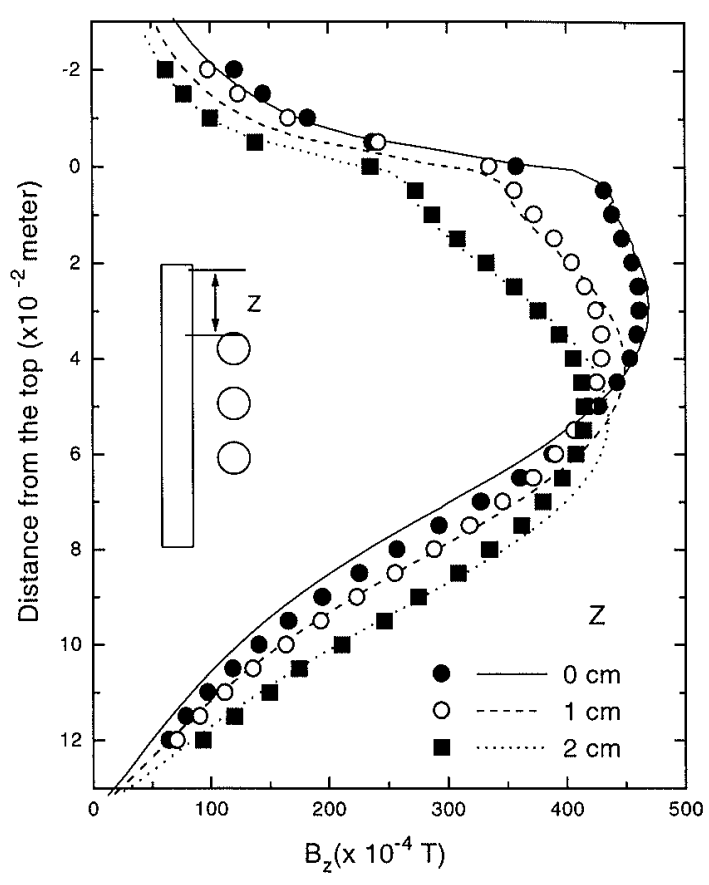

Fig. 3. Verification of electromagnetic calculation. line: calculation, symbol: experiment

Faraday's law;

$$
B_{z}=\frac{\Phi_{\mathrm{pp}}}{4 \sqrt{2} \pi^{2} \cdot a^{2} \cdot f \cdot n}
$$

The meniscus shape of molten tin in the cold crucible was also measured using a needle type probe made of stainless rod of $2 \mathrm{~mm} \phi$ and the $3 \mathrm{D}$ translation device.

The calculated magnetic flux density distribution along the $z$-axis at the center of the cold crucible agrees very well with the measured values in the empty crucible as shown in Fig. 3.

\subsection{Verification of Calculation of Meniscus Shape}

In order to predict the shape of molten metal in a cold crucible, the magnetic pressure distribution has to be obtained. Figure 4 shows the calculated and measured shapes of molten tin in the continuous casting type cold crucible. They agrees rather well with each other except at the contact region between the melt and inner surface of the crucible.

Fukumoto et al. $^{20)}$ reported that when the frequency is low (around $1 \mathrm{kHz}$ ) the velocity field is not too small to be neglected. However, in this system the applied current frequency is relatively high $(5-9.6 \mathrm{kHz})$ so that the effect of the velocity field becomes insignificant as the frequency increases. It is concluded from the results that at $6.5 \mathrm{kHz}$ or higher frequency, one can predict the meniscus shape with reasonable accuracy without considering the actual flow field distribution. Discrepancy between the calculation and the experiments near the contact region is thought to be corresponding to the effects of dynamic pressure and oxide film of the charge.

\subsection{Verification of Fluid Flow Model}

In order to examine validation of developed BFC code in calculating fluid flow and heat transfer, comparison between results obtained from developed code MPLGC and commercial CFD (Computational Fluid Dynamics) 
code PHOENICS (owned by POSCO) on the free convection problem in the cavity was conducted. The test system shown in Fig. 5 has hot wall (left side wall), cold wall (right side wall) and two adiabatic walls (top and bottom walls) and the difference of temperature between hot and cold wall is set to $1{ }^{\circ} \mathrm{C}$. Dimension of this tapered cavity is $0.1 \mathrm{~m} \times(0.05-0.1) \mathrm{m}$ and $20 \times 20$ non-orthogonal grid system is used to include the non-orthogonal source calculation. Figure 5 shows the velocity vector field in the system.

The results show good agreements between the two codes. The maximum velocity obtained from MPLGC and PHOENICS are 1.974E-5 m/sec, $1.964 \mathrm{E}-5 \mathrm{~m} / \mathrm{sec}$, respectively. The difference is only $0.5 \%$. Figure 6 shows the comparisons of the presently predicted covarient velocity profiles at $x=1 / 2 L_{x}$ with the predictions by PHOENICS. The results of MPLGC match excellently with that of PHOENICS in spite of the fact that developed code uses non-staggered and PHOENICS uses staggered grid system. Corresponding to the checker-

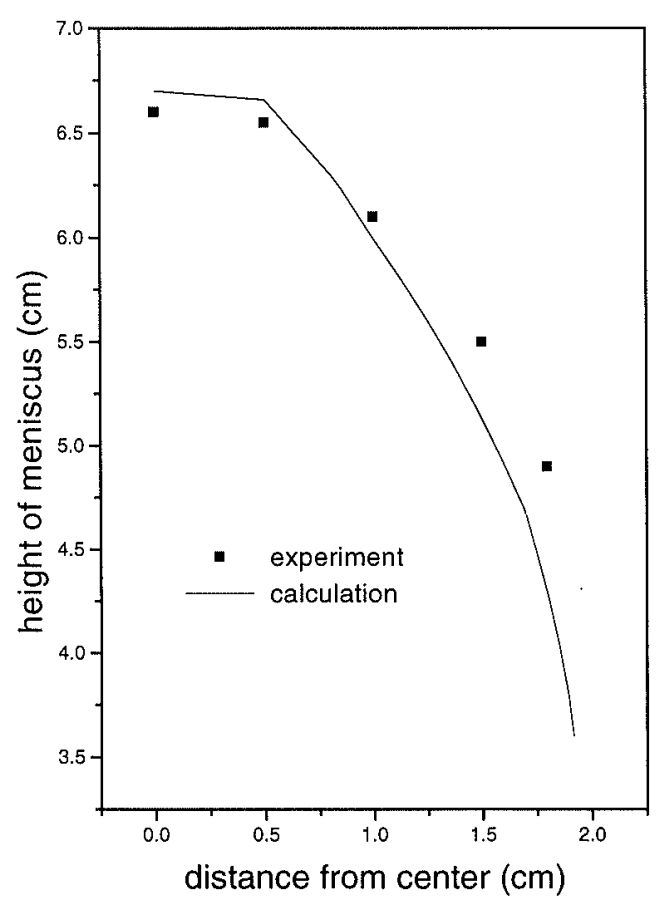

Fig. 4. Verification of meniscus shape calculation. board phenomenon, the distribution of pressure obtained from several cases of the calculation of cold crucible was examined, but no checkerboard pheonomenon was found.

\section{Results and Discussion}

\subsection{Fluid Flow and Heat Transfer in the Cold Crucible}

Figure 7 shows the calculated result of various physical phenomena occuring in the half segment of cold crucible when electric current of $470 \mathrm{~A}$ and $6.5 \mathrm{kHz}$ is applied to coil.

Joule heating and magnetic force distribution in the cold crucible is presented in Fig. 7(a). Here, contour lines represent the distribution of Joule heating and the vectors show electromagnetic force. Because of high frequency, magnetic force as well as Joule heating is concentrated on the surface. The magnitude of magnetic force is largest near the solidification front and decays as the height increases. Due to such a distribution of magnetic force, unequally pressed molten tin forms a circulation flow. Figure 7(b) represents the fluid motion and the solidification line in the charge. The thick line and the dotted line represent solidification fronts obtained by calculation and experiments, respectively. The heat source is Joule heating, and the heat sink is radiation and conduction by the contact between charge and crucible wall. Because of the magnetic pressure and possible oxide film of the charge, the contact between charge and crucible wall is not complete. Shrinkage by the solidification also increase the incompleteness of contact. To consider the effect of this incomplete contact, a coefficient named contact ratio is multiplied to the heat transfer coefficient. Figure 7 is obtained under the assumption that the contact ratio is 0.2 . With this contact ratio of 0.2 , the calculated and the experimental measurements agree well each other. This means that the heat flux through the mold in electromagnetically confined casting system is much less than that of complete contact. Contact ratio is merely a constant, not a function. More study will be required to make it a function of material properties, current, frequency, etc. The maximum velocity of the flow is about $0.17 \mathrm{~m} / \mathrm{sec}$ and is formed beneath the meniscus. This downward flow have

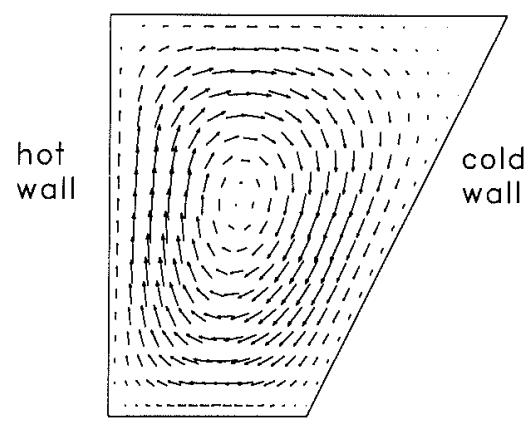

(a)

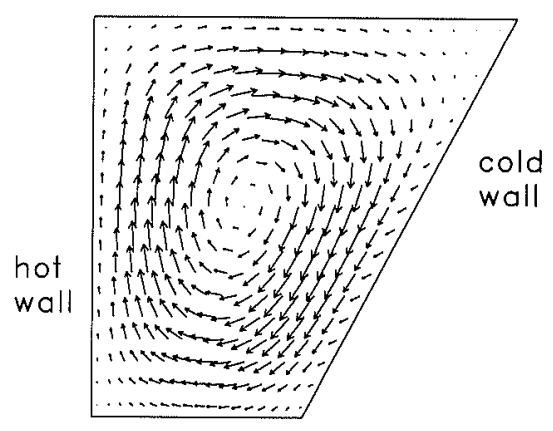

(b)

Fig. 5. Velocity field obtained from two CFD code

(a) Code developed in this study (MPLGC)

(b) Commercial CFD code PHOENICS 


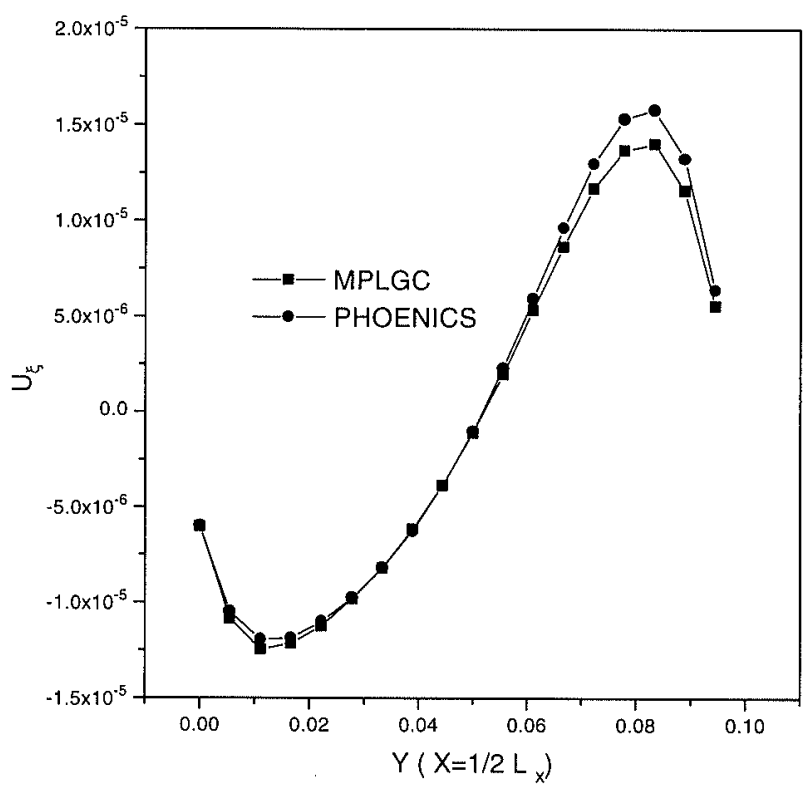

Fig. 6. Comparison of velocity profile $\left(X=1 / 2 L_{x}\right)$.

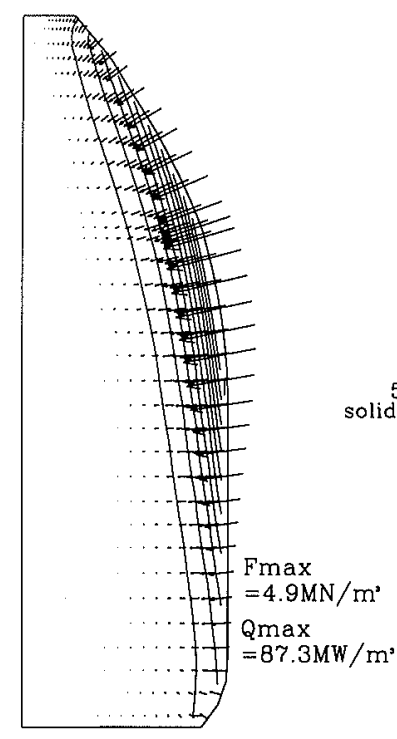

(a)

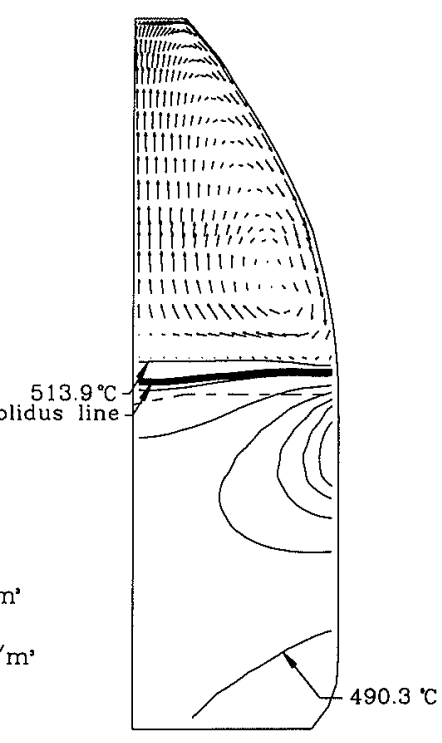

(b)
Fig. 7. Calculated results obtained with electric current of $470 \mathrm{~A}$ and $6.5 \mathrm{kHz}$.

(a) Joule heating and electromagnetic body force

(b) Fluid flow, temperature distribution and solidification

an effect preventing the formation of concave type solidification front by supplying relatively hot melt at the surface to the contact region. The circulation flow sweeps the surface of the solid-liquid interface and make it smooth. The temperature distribution in the melt become uniform by circulating flow.

Figure 8 shows the distribution of temperature profile and fluid flow field obtained with the fictitious contact ratio of 0.15 (Fig. 8(a)) and 0.25 (Fig. 8(b)) respectively. Thick line and dotted line also represent solidification fronts obtained by calculation and experiment, respectively. Figure 8(a) corresponds to the case of contact ratio 0.15 . In this figure, the underestimation of heat flux through conduction region lowers calculated

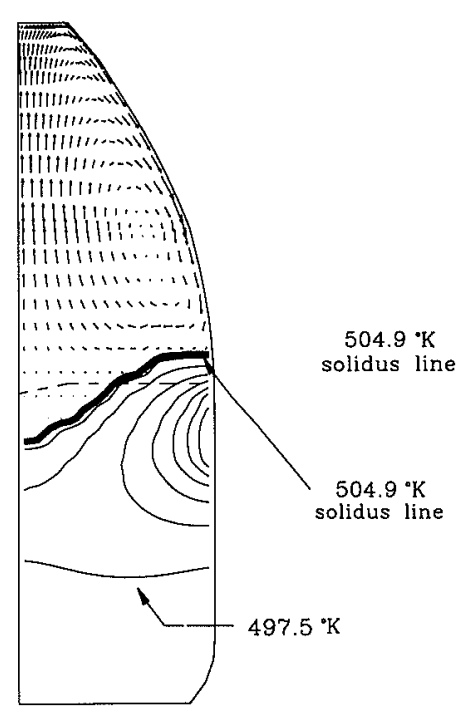

(a)

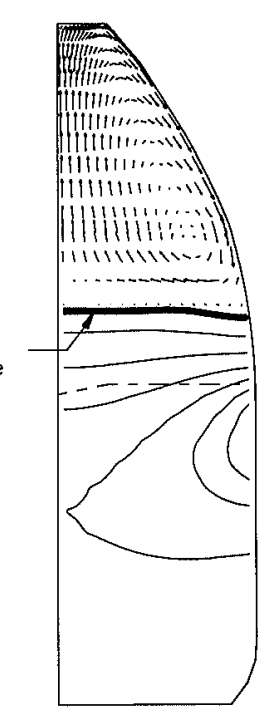

(b)
Fig. 8. Fluid flow and solidification with varied contact ratio. (a) Contact ratio $=0.15$

(b) Contact ratio $=0.25$

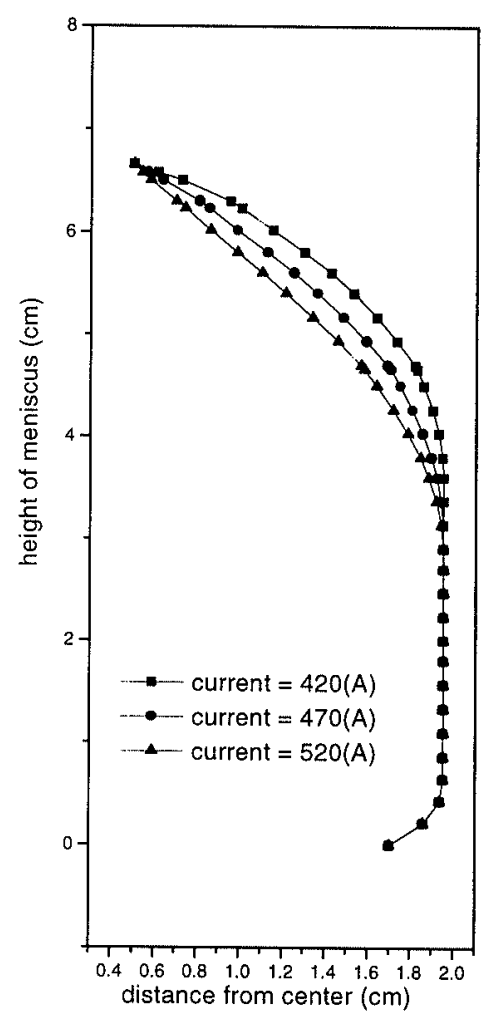

Fig. 9. Influence of coil current on meniscus shape.

solidification front below the experimental contour lines. On the other hand, overestimation of conducting heat transfer lifts the solidification front over the experimental contour line. With the contact ratio of 0.20 the solidus line is well matched with experiments. Therefore, it can be mentioned that conducting heat flux near the solidification front is about $20 \%$ of complete contacting of common casting. 


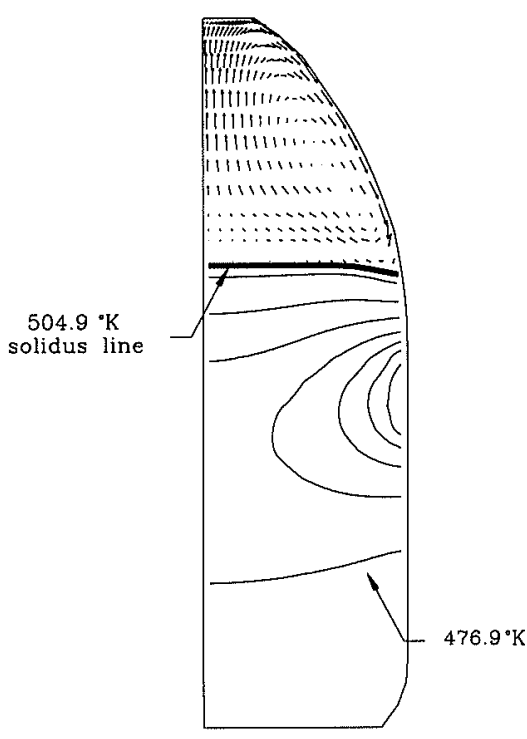

(a)

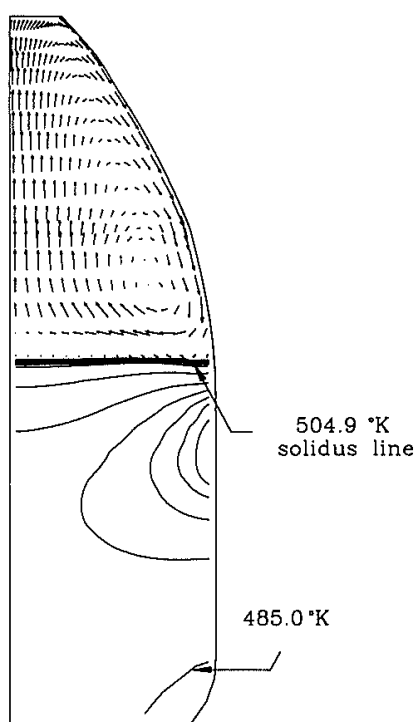

(b)

Fig. 10. Fluid flow and solidification with varied operating parameters.

(a) Coil current $=420 \mathrm{~A}$, frequency $=6.5 \mathrm{kHz}$

(b) Coil current $=470 \mathrm{~A}$, frequency $=6 \mathrm{kHz}$

\subsection{Influence of Coil Current on Meniscus Shape and Solidification Front}

Influence of coil current on the calculated meniscus shape is shown in Fig. 9. Magnetic pressure acting on the meniscus is known to be proportional to the square root of coil current. In this calculation, noticeable change of the meniscus shape could be obtained with the small change of the applied current of about $50 \mathrm{~A}$.

Figure 10 shows the fluid flow and solidification status of cold crucible with varied operating parameter. In the case of Fig. 10(a), coil current is decreased to $420 \mathrm{~A}$ with same frequency condition. The result obtained with varied frequency is shown in Fig. 10(b). It can be seen that about $10 \%$ decrease of the applied current moves solidification front upward significantly. With the $7 \%$ decrease in frequency, there is no noticeable change in location of solidification front. The maximum velocity is decreased to 0.141 from $0.167 \mathrm{~m} / \mathrm{sec}$ corresponding to a $10 \%$ decrease in applied current. When magnitude of current is increased to 520 from $470 \mathrm{~A}$, maxmum velocity is also increased to $0.181 \mathrm{~m} / \mathrm{sec}$. These effects are larger than that caused by frequency changes. With $7 \%$ of decreased frequency, difference of maximum velocity is only about $0.001 \mathrm{~m} / \mathrm{sec}$. This is considered to be the cancelling effect of skin depth. Though magnetic force decreases with reduced frequency, increased skin depth prevents the decrease of velocity. So velocity of fluid flow is seen to be less affected by variation of frequency.

\section{Conclusion}

An advanced mathematical model was developed that could calculate fluid flow and solidification, taking into consideration for 3D shape of cold crucible. This model consumes less computing resources by effective algo- rithm. Validity of the model was confirmed by experimental measurements of solidification front and meniscus shape and by commercial CFD code. From a series of numerical study using this model, the following results are obtained.

(1) Unequal distribution of the magnetic force induces recirculation flow in the melt. Temperature distribution in the melt becomes uniform due to this fluid flow and shape of the solidification front is also affected by this flow.

(2) Velocity of this flow is influenced by magnitude and frequency of the applied current. Influence of the magnitude of coil current is more dominant.

(3) Shape of the solidification is well predicted by the mathematical model. In the course of calculation, it is known that contact between charge and crucible wall is incomplete near the solidification front. And heat flux in the cold crucible used in this study is as small as $20 \%$ of that of complete contacting conducting heat transfer.

(4) By using $\mathrm{H}-\phi$ method and BFC with nonstaggered system, an effective simulation model was developed.

\section{Acknowledgement}

The authors would like to thank POSCO for the support during the period of this work.

\section{Nomenclature}

$a:$ radius of sensor coil

$B$ : magnetic flux density

$B_{i}$ : magnetic flux density components

$B_{z}$ : magnetic flux density in $z$ direction

$E$ : electric field intensity

$G^{i}$ : flux components defined on the faces of control colvume

$g^{i j}$ : contravariant metric tensor 
$H$ : magnetic field intensity $\left(H=H_{0}+H_{r}\right)$

$H_{0}$ : magnetic field intensity due to $J_{0}$

$H_{r}$ : magnetic field intensity generated by induced eddy current

$H_{\tau}$ : tangential magnetic field intensity on the meniscus of charge

$h$ : heat transfer coefficient

$J$ : Jacobian of the coordinate transformation

$J_{0}$ : current applied to the induction coil

$J_{\mathrm{e}}:$ eddy current

$n$ : turns of sensor coil

$n_{\mathrm{c}}$ : unit outward normal vector from conductor

$n_{\mathrm{nc}}$ : unit outward normal vector from non-conducting region

$P_{\mathrm{g}}:$ hydrostatic pressure

$P_{\mathrm{m}}$ : magnetic pressure

$P_{\mathrm{s}}$ : pressure due to surface tension

$Q$ : Joule heat per unit volume

$R_{1}, R_{2}$ : principal curvatures of meniscus

$S$ : boundary surface between conducting and non-conducting region

$S_{\phi}$ : source term in general governing equation

$T:$ temperature

$T_{0}$ : temperature of surroundings

$u_{i}:$ velocity components

$V_{\mathrm{c}}$ : volume of the conducting region

$V_{\mathrm{nc}}$ : volume of the non-conducting region

$w$ : scalar weighting function

$W$ : vector weighting function

$x_{i}$ : axes of cartesian coordinates

$z$ : axis of axial direction

$\Gamma: \quad$ surface tension coefficient

$\Gamma_{\phi}:$ diffusion coefficient in general governing equation

$\delta:$ skin depth

$\mu: \quad$ magnetic permeability

$\mu_{1}:$ laminar viscosity

$\mu_{\mathrm{t}}$ : turbulent viscosity $\mu_{\text {eff }}$ : effective viscosity $\left(\mu_{\text {eff }}=\mu_{1}+\mu_{\mathrm{t}}\right)$

$\xi_{i}$ : axes of curvilinear coordinates

$\rho:$ density

$\sigma:$ electrical conductivity

$\Phi_{\mathrm{pp}}$ : amplitude of alternating voltage induced in sensor coil (V)

$\phi$ : magnetic scalar potential

\section{REFERENCES}

1) T. Tanaka, K. Kurita and A. Kuroda: ISIJ Int., 31 (1991), 350.

2) Y. Kawase, T. Yamaguchi and N. Hayashi: IEEE-Trans. Magnetics, 29 (1993), 1554.

3) H. Tsuboi, M. Tanaka, F. Kobayashi and T. Misaki: LEEE-Trans. Magnetics, 29 (1993), 1574.

4) A. Gagnoud and J. P. Brancher: IEEE-Trans. Magnetics, 21 (1985), 2424.

5) W. R. Smythe: Static and Dynamic Electricity, 3rd Ed., McGraw-Hill, New York, (1993), 290.

6) A. Gagnoud and I. Leclercq: IEEE-Trans. Magnetics, 24 (1988), 573.

7) K. Iwai, K. Sassa and S. Asai: Tetsu-to-Hagané, 79 (1993), 1053.

8) H. Makino, M. Kuwabara and S. Aasi: Tetsu-to-Hagané, 81 (1995), 523

9) T. Tanaka, K. Kurita and A. Kuroda: ISIJ Int., 31 (1991), 1416.

10) J. P. Webb and B. Forghani: IEEE-Trans. Magnetics, 29 (1993), 2461.

11) S. V. Patankar: Numerical Heat Transfer and Fluid Flow, McGraw-Hill, New York, (1980), 116.

12) A. W. Date: Int. J. Heat Mass Transfer, 36 (1993), 1913.

13) Y. W. Cho. Y. J. Oh, K. W. Yi, S. H. Chung and J. D. Shim: Modelling and Simulation in Materials Science and Engineering (to be published).

14) A. Bossavit: IEEE Proc., 135 (1988), 493.

15) Kim, Kyung Yup: Ph. D. Dissertation, Seoul National Univ., (1993).

16) C. M. Rhie and W. L. Chow: AIAA J., 21 (1983), 1525.

17) C. J. Kim and S. T. Ro: Numer. Heat Transfer, 27 (1995), 355.

18) J. O. Hinze: Turbulence, McGraw Hill, New York, (1975).

19) W. Kurz and D. J. Fisher: Fundamentals of Solidification, 3rd ed., Trans. Tech. Pub., USA, (1989).

20) H. Fukumoto, Y. Hosokawa, K. Ayata and M. Morishita: 1991 Magnetohydrodynamics in Process Metallurgy, ed. by J. Szekely et al., The Minerals, Metals and Meterials Society, Warrendale, Pennsylvania, 21. 